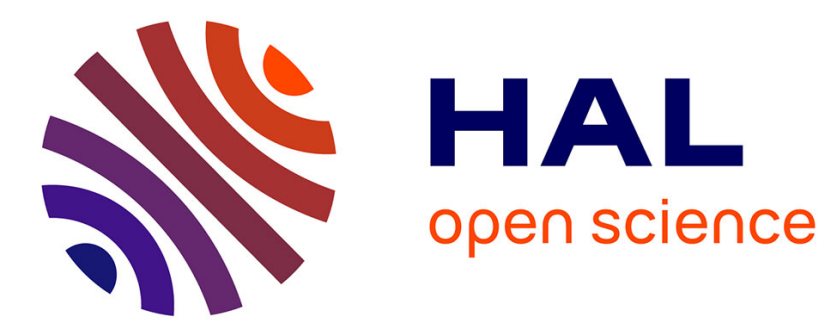

\title{
Real wave vectors for dynamic analysis of periodic structures
}

\author{
Angelo Luongo, Francesco Romeo
}

\section{To cite this version:}

Angelo Luongo, Francesco Romeo. Real wave vectors for dynamic analysis of periodic structures. Journal of Sound and Vibration, 2005, 279 (1-2), pp.309-325. hal-00794673

\section{HAL Id: hal-00794673 \\ https://hal.science/hal-00794673}

Submitted on 26 Feb 2013

HAL is a multi-disciplinary open access archive for the deposit and dissemination of scientific research documents, whether they are published or not. The documents may come from teaching and research institutions in France or abroad, or from public or private research centers.
L'archive ouverte pluridisciplinaire HAL, est destinée au dépôt et à la diffusion de documents scientifiques de niveau recherche, publiés ou non, émanant des établissements d'enseignement et de recherche français ou étrangers, des laboratoires publics ou privés. 


\title{
Real wave vectors for dynamic analysis of periodic structures
}

\author{
A. Luongo ${ }^{\mathrm{a}, *}$, F. Romeo ${ }^{\mathrm{b}}$ \\ a Dipartimento di Ingegneria delle Strutture, delle Acque e del Terreno, Università di L'Aquila, \\ Monteluco di Roio L'Aquila 67040, Italy \\ ${ }^{\mathrm{b}}$ Dipartimento di Ingegneria Strutturale e Geotecnica, Università di Roma "La Sapienza", \\ Via Gramsci 53,197 Roma, Italy
}

\begin{abstract}
A modified version of the traditional wave vector computational scheme for the dynamic analysis of long undamped periodic structures is presented. First, the consistency of the complex wave vector mathematical formulation is discussed, placing particular emphasis on the real or complex nature of the resulting characteristic equation from which the natural frequencies are derived. It is shown that the rearrangement in terms of complex waves entering the domain, devised to overcome ill-conditioning arising in the transfer matrix formulation, entails as a side effect an ill-posed problem, as it leads to a complex characteristic equation in a real unknown. Next, the proposed approach is described. It provides for transformation of frequency-dependent real transfer matrices for state vectors to real transfer matrices for wave vectors, thus avoiding an ill-conditioned and ill-posed problem. Finally, applications to mono-coupled and bi-coupled structures are illustrated, aiming at comparing the proposed method with the transfer matrix and complex wave vector approaches.
\end{abstract}

\section{Introduction}

The well-known wave vector approach has been proposed [1,2] for the analysis of the response of long undamped periodic structures to overcome numerical difficulties arising in the transfer matrix formulation when the number of periodic units increases. Its effectiveness has been confirmed by a number of applications pertaining to trusses [3,4], generic structural networks [5], wave localization phenomena [6] and piecewise periodic structures [7]. Nevertheless, a number of authors have encountered numerical difficulties to determine the natural frequencies when solving

*Corresponding author. Tel.: + 39862 434511; fax: + 39862434548.

E mail addresses: luongo@ing.univaq.it (A. Luongo), francesco.romeo@uniroma1.it (F. Romeo). 
the complex determinant resulting from the wave vector approach. For finite truss beams, Pierre and Chen [8] ascribed such difficulties to the high modal density. They observed that the zeros of the real and imaginary parts of the characteristic equation were not even close enough to derive natural frequencies yielding accurate mode shapes; to circumvent the problem they transformed the latter determinant into a real one. Moreover, Signorelli and von Flotow [3] considered the absolute value of the complex characteristic determinant stating that the natural frequencies of the truss need to be identified whenever it "tends" to vanish.

In this work, the causes of such drawbacks are investigated. It is shown that numerical difficulties are due to the ill-posed mathematical problem in terms of complex waves incoming in the structure. In fact, according to this method, natural frequencies are found as real solutions of complex characteristic equations, i.e. as a solution of a system of two equations in just one unknown. Aiming to overcome such problems while retaining the advantages of the wave transfer matrix framework, a modified version of the traditional wave vector computational scheme is proposed. The method deals with real quantities only, thus avoiding the described pathology. In particular, while the traditional method implies the transformation of frequency-dependent real transfer matrices for state vectors to complex transfer matrices for wave vectors, the approach proposed here provides for transformation to real matrices. In essence the computational scheme adopts a basis of eigenvectors coinciding with the real and imaginary parts of the transfer matrix complex eigenvectors thereby leading to a real block-diagonal matrix. The blocks associated with eigenvalues with modulus greater than one are inverted, while those associated with unit modulus eigenvalues are left unaltered because they do not feed ill-conditioning. As a consequence a two-fold objective is pursued. On one hand, the obtained real boundary condition's matrix is more suited for free vibration analysis; on the other hand, the stability of computations, assured by proceeding in the direction of wave motion, is preserved. The proposed transformation can also be interpreted as a change of co-ordinates that brings the linear map $\mathbf{x}_{k+1}=\mathbf{T} \mathbf{x}_{k}$ into a normal form, $\mathbf{x}_{k}$ being the state vector at the coupling point $k$ and $\mathbf{T}$ the transfer matrix.

The paper is organized as follows. In Section 2 the mechanism leading to an ill-posed problem is discussed in a broader context, by dealing with steady-waves analysis of an axially vibrating rod. The proposed real wave vector approach is described in Section 3 for generic $n$-coupled periodic structures. By comparing this approach with the earlier one [1,2], whose main steps are outlined in the appendix, the differences between the two algorithms are highlighted. Afterwards, in Section 4 , an illustrative analytical application to a mono-coupled mass spring chain is presented. Finally, numerical simulations pertaining free and forced vibration analysis of bi-coupled periodic beams resting on elastic supports are reported in Section 5. Throughout all the examples the emphasis is placed on the comparison among the features of the transfer matrix, complex and real wave vector approaches.

\section{An introductory problem: real and complex formulations}

With the aim of introducing the problem, consider the free axial vibrations of a free free rod of length $l$. They are governed by the following boundary value problem: 
Standing waves in a free free rod

\begin{tabular}{|c|c|c|}
\hline Solution & Boundary conditions & Characteristic equation \\
\hline \multicolumn{3}{|l|}{ Real form } \\
\hline $\begin{array}{l}u=a \cos \beta x+b \sin \beta x \\
a, b \in \mathbb{R}\end{array}$ & {$\left[\begin{array}{cc}0 & 1 \\
\sin \beta l & \cos \beta l\end{array}\right]\left(\begin{array}{l}a \\
b\end{array}\right)=0$} & $\sin \beta l=0$ \\
\hline$\left(a_{1}\right)$ & $\left(b_{1}\right)$ & $\left(c_{1}\right)$ \\
\hline \multicolumn{3}{|l|}{ Complex form } \\
\hline $\begin{array}{l}u=r_{A} \mathrm{e}^{\mathrm{i} \beta x}+l_{A} \mathrm{e}^{-\mathrm{i} \beta x} \\
r_{A}, l_{A} \in \mathbb{C}\end{array}$ & {$\left[\begin{array}{cc}1 & 1 \\
\mathrm{e}^{\mathrm{i} \beta l} & \mathrm{e}^{-\mathrm{i} \beta l}\end{array}\right]\left(\begin{array}{l}r_{A} \\
l_{A}\end{array}\right)=0$} & $\mathrm{e}^{\mathrm{i} \beta l} \quad \mathrm{e}^{-\mathrm{i} \beta l}=0$ \\
\hline$\left(a_{2}\right)$ & $\left(b_{2}\right)$ & $\left(c_{2}\right)$ \\
\hline Modified complex form & & \\
\hline $\begin{array}{l}u=r_{A} \mathrm{e}^{\mathrm{i} \beta x}+l_{B} \mathrm{e}^{-\mathrm{i} \beta(x-l)} \\
r_{A}, l_{B} \in \mathbb{C}\end{array}$ & {$\left[\begin{array}{cc}1 & \mathrm{e}^{\mathrm{i} \beta l} \\
\mathrm{e}^{\mathrm{i} \beta l} & 1\end{array}\right]\left(\begin{array}{l}r_{A} \\
l_{B}\end{array}\right)=0$} & $1=0$ \\
\hline$\left(a_{3}\right)$ & $\left(b_{3}\right)$ & $\left(c_{3}\right)$ \\
\hline
\end{tabular}

$$
\begin{aligned}
& u^{\prime \prime}(x)+\beta^{2} u(x)=0 \quad x \in[0, l], \\
& u^{\prime}(0)=0, \quad u^{\prime}(l)=0,
\end{aligned}
$$

in which $\beta$ is the eigenvalue, proportional to the time frequency $\omega$. Solutions to Eq. (1) can be put in different forms, here named real, complex and modified complex forms, summarized in Table 1. If the field $u(x)$ is expressed in the real form (equation $\left(a_{1}\right)$ ), the boundary conditions ( $\left.1 \mathrm{~b}\right)$ lead to the real eigenvalue problem $\left(b_{1}\right)$, whose real characteristic equation $\left(c_{1}\right)$ admits the roots:

$$
\vartheta=\frac{j \pi}{N} \text { with } j=0,1, \ldots \text {. }
$$

Alternatively, the solution can be expressed in the complex form $\left(a_{2}\right)$. In it, $\mathrm{e}^{\mathrm{i} \beta x}$ and $\mathrm{e}{ }^{\mathrm{i} \beta x}$ are steady waves (conventionally) propagating rightward and leftward respectively. Their complex amplitudes, $r_{A}$ and $l_{A}$, represent the contribution of each wave to the displacement at the left end $A$ of the road, where $\mathrm{e}^{\mathrm{i} \beta x}$ and $\mathrm{e}^{\mathrm{i} \beta x}$ assume unit values. By enforcing boundary conditions, the equations $\left(b_{2}\right)$ are drawn. In them, the columns of the matrix are linear combinations of the columns of the matrix in equation $\left(b_{1}\right)$, hence the same real characteristic equation $\left(c_{2}\right)$ follows. Therefore, the real and complex forms are equivalent. Finally, a modified complex form of the solution is considered (equation $\left(a_{3}\right)$ ), derived from equation $\left(a_{2}\right)$ by letting $l_{A}:=l_{B} \mathrm{e}^{\mathrm{i} \beta l}$. This change in the complex amplitudes is often performed in order to avoid numerical ill-conditioning in the boundary conditions when $l$ is large and $\beta$ is allowed to assume complex values (e.g. for long beams resting on Winkler soil, or for long tubes with radially symmetric loads). In fact, the modification renders complex amplitudes of the same order of magnitude otherwise very different from each other. By again enforcing boundary conditions, equations $\left(b_{3}\right)$ are drawn. They could be obtained directly from equations $\left(b_{2}\right)$ through the product of the second column of the matrix by the factor $\mathrm{e}^{\mathrm{i} \beta l}$, which transforms $l_{A}$ into $l_{B}$. Since the factor is complex, the associated 
characteristic equation $\left(c_{3}\right)$, differently from the previous cases, is also complex. It leads to two real equations in the unique real unknown $\beta$, namely:

$$
\cos 2 \beta l=1, \quad \sin 2 \beta l=0 .
$$

Problem (3) appears as an overdetermined non-linear system, since it requires satisfying two simultaneous equations with just one unknown. It will be referred to as ill-posed, since the true nature of the determined system is disguised. In principle, no solutions exist for such a problem; however, it is easy to check that Eqs. (3) do admit solutions, and these all coincide with the solutions (2). This circumstance is a consequence of the fact that, since a column of the matrix in equation $\left(b_{2}\right)$ has been altered by $\mathrm{e}^{\mathrm{i} \beta l}$, also its characteristic equation $\left(c_{2}\right)$ has been multiplied by the same factor, so that equation $\left(c_{3}\right)$ admits the factorized form:

$$
\mathrm{e}^{\mathrm{i} \beta l}\left(\mathrm{e}^{\mathrm{i} \beta l} \quad \mathrm{e}^{\mathrm{i} \beta l}\right)=0 .
$$

Since the factor never vanishes, Eq. (4) admits the same roots of equation $\left(c_{2}\right)$ and no other ones. However, in more complex cases than that at hand, it is not easy to factorize the characteristic equation, and one has to solve a problem of the following form:

$$
f(\omega)+\mathrm{i} g(\omega)=0,
$$

where $\omega$ is the unknown. There are two ways to solve this equation: (a) to search for complex roots $\omega=\gamma+\mathrm{i} \delta$ of the simultaneous equations $\operatorname{Re}(f) \quad \operatorname{Im}(g)=0, \operatorname{Im}(f)+\operatorname{Re}(g)=0$, by retaining only the real solutions $\gamma \neq 0, \delta=0$; (b) to search for real roots $\omega$ of each equation $f(\omega)=0$ and $g(\omega)=0$, by keeping only the common roots. Numerical problems arise in both the approaches, since it is difficult to decide whether roots with small $\gamma$ 's (in the approach (a)) or roots very close to each other (in the approach (b)) must be retained or rejected. Such difficulties were encountered in the works cited in the Introduction, as a consequence of the ill-position of the problem.

In conclusion, the complexity of the characteristic equation does not depend on the complexity of the amplitudes, but rather on their rearrangement. The expedient of the change of the variables is a peculiar aspect of the complex wave coordinate method; it permits the avoidance of numerical ill-conditioning, but entails an ill-posed problem. In the next section a variant of the method is illustrated, able to avoid an ill-conditioned and ill-posed problem.

\section{Real wave vector approach}

For periodic structures, the state vectors $\mathbf{x}_{k}=\left(\mathbf{d}_{k}, \mathbf{f}_{k}\right)$, listing generalized displacements $\mathbf{d}$ and forces $\mathbf{f}$ at the coupling point $k$, is related to the state vector $\mathbf{x}_{k+1}$, at the following coupling point $k+1$, through the transfer matrix $\mathbf{T}$, i.e. $\mathbf{x}_{k+1}=\mathbf{T} \mathbf{x}_{k}$. For elements coupled through $n$ degrees of freedom, $\mathbf{T}$ is a real $2 n \times 2 n$ frequency dependent matrix and, if the structure is composed by $N$ elements connecting nodes $A$ and $B$, then

$$
\left(\begin{array}{c}
\mathbf{d}_{B} \\
\mathbf{f}_{B}
\end{array}\right)=\mathbf{T}^{N}\left(\begin{array}{c}
\mathbf{d}_{A} \\
\mathbf{f}_{A}
\end{array}\right) .
$$




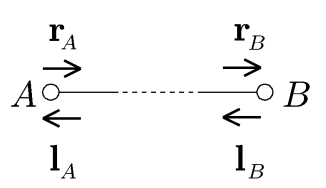

(a)

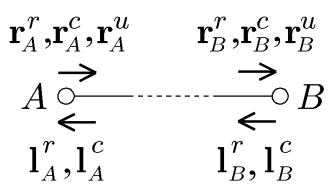

(b)

Fig. 1. Wave vector components: (a) complex; (b) real.

A peculiar property of the transfer matrix is that its eigenvalues are reciprocal pairs $(\lambda, 1 / \lambda)$. The case of distinct eigenvalues will be considered throughout the analysis. According to the wellknown complex wave vectors approach (see the appendix), the state vectors can be transformed to complex wave vectors through a matrix whose columns are the complex eigenvectors of $\mathbf{T}$. The obtained wave vector $\left(\mathbf{r}_{k}, \mathbf{l}_{k}\right)^{\mathrm{T}}$, at the generic coupling point $k$, has components $\mathbf{r}$ and $\mathbf{I}$ hinting at right-going and left-going waves, respectively (see Fig. 1(a)). They represent the amplitudes of the eigenvectors associated to reciprocal pairs with $|\lambda|<1$ and $|\lambda|>1$, respectively; if $|\lambda|=1$, the two reciprocal eigenvalues are complex conjugate and the associated amplitudes split in $\mathbf{r}$ and $\mathbf{l}$.

An alternative transformation can be adopted relying on a modified basis of eigenvectors. Consider the generic case of $\mathbf{T}$ with $2 n_{r}$ real eigenvalues $\lambda=\rho, 1 / \rho, 2 n_{c}$ complex eigenvalues $\lambda=\rho \mathrm{e}^{ \pm \mathrm{i} \theta}, 1 / \rho \mathrm{e}^{\mp \mathrm{i} \theta}$ with $\rho<1$ and $2 n_{u}$ complex eigenvalues with unit modulus $\lambda=\mathrm{e}^{ \pm \mathrm{i} \theta}$. Let also $\mathbf{u}_{h}$ and $\mathbf{u}_{h}^{*}, h=1, \ldots, n_{r}$, be the real eigenvectors of $\mathbf{T}$ associated with the real eigenvalues $\rho_{h}$ and $1 / \rho_{h}$, respectively; $\mathbf{z}_{j}$ and $\mathbf{z}_{j}^{*}, j=1, \ldots, n_{c}$, be the complex eigenvectors associated with the eigenvalues $\rho_{j} \mathrm{e}^{ \pm \mathrm{i} \theta_{j}}, 1 / \rho_{j} \mathrm{e}^{\mp \mathrm{i} \theta_{j}}$, respectively; $\mathbf{y}_{l}, l=1, \ldots, 2 n_{u}$, be the complex eigenvectors associated with the eigenvalues $\mathrm{e}^{ \pm \mathrm{i} \theta_{l}}$. By setting up the invertible matrix $\mathbf{U}$ in the form

$$
\mathbf{U}=\left[\mathbf{u}_{h}, \operatorname{Re}\left(\mathbf{z}_{j}\right), \operatorname{Im}\left(\mathbf{z}_{j}\right), \operatorname{Re}\left(\mathbf{y}_{l}\right), \operatorname{Im}\left(\mathbf{y}_{l}\right), \mathbf{u}_{h}^{*}, \operatorname{Re}\left(\mathbf{z}_{j}^{*}\right), \operatorname{Im}\left(\mathbf{z}_{j}^{*}\right)\right]
$$

it is well known that it is possible to obtain a matrix

$$
\mathbf{U}^{1} \mathbf{T} \mathbf{U}=\operatorname{diag}\left(\lambda_{h}, \mathbf{C}_{j}, \mathbf{P}_{l}, \lambda_{h}{ }^{1}, \mathbf{C}_{j}{ }^{1}\right),
$$

representing a real transfer matrix for wave vectors. In Eq. (8) $\mathbf{C}_{j}, 1 \leqslant j \leqslant n_{c}$, are $2 \times 2$ blocks:

$$
\mathbf{C}_{j}=\rho_{j}\left[\begin{array}{cc}
\cos \theta_{j} & \sin \theta_{j} \\
\sin \theta_{j} & \cos \theta_{j}
\end{array}\right],
$$

and $\mathbf{P}_{l}, 1 \leqslant l \leqslant 2 n_{u}$, are submatrices equal to $\mathbf{C}_{j}$ with $\rho=1$. The state vector transformation can now be expressed through a new change of co-ordinates to real wave vector using the real matrix $\mathbf{U}$ as

$$
\left(\begin{array}{l}
\mathbf{d}_{k} \\
\mathbf{f}_{k}
\end{array}\right)=\mathbf{U}\left(\mathbf{r}_{k}^{r}, \mathbf{r}_{k}^{c}, \mathbf{r}_{k}^{u}, \mathbf{l}_{k}^{r}, \mathbf{l}_{k}^{c}\right)^{\mathrm{T}},
$$

where the real wave vector at node $k$ has been partitioned into five subvectors $\mathbf{r}_{k}^{r}, \mathbf{r}_{k}^{c}, \mathbf{r}_{k}^{u}, \mathbf{r}_{k}^{r}$ and $\mathbf{l}_{k}^{c}$, representing the amplitudes of the eigenvectors $\mathbf{u}_{h}, \mathbf{z}_{j}, \mathbf{y}_{l}, \mathbf{u}_{h}^{*}$ and $\mathbf{z}_{j}^{*}$, respectively (see Fig. 1(b)). 
Substituting Eq. (10) into Eq. (6) gives

$$
\left(\begin{array}{c}
\mathbf{r}_{B}^{r} \\
\mathbf{r}_{B}^{c} \\
\mathbf{r}_{B}^{u} \\
\mathbf{r}_{B}^{r} \\
\mathbf{l}_{B}^{c}
\end{array}\right)=\left[\begin{array}{ccccc}
\mathbf{S}^{N} & \mathbf{0} & \mathbf{0} & \mathbf{0} & \mathbf{0} \\
\mathbf{0} & \mathbf{C}^{N} & \mathbf{0} & \mathbf{0} & \mathbf{0} \\
\mathbf{0} & \mathbf{0} & \mathbf{P}^{N} & \mathbf{0} & \mathbf{0} \\
\mathbf{0} & \mathbf{0} & \mathbf{0} & \mathbf{S}^{N} & \mathbf{0} \\
\mathbf{0} & \mathbf{0} & \mathbf{0} & \mathbf{0} & \mathbf{C}
\end{array}\right]\left(\begin{array}{c}
\mathbf{r}_{A}^{r} \\
\mathbf{r}_{A}^{c} \\
\mathbf{r}_{A}^{u} \\
\mathbf{l}_{A}^{r} \\
\mathbf{l}_{A}^{c}
\end{array}\right),
$$

where the square submatrices in the global wave transfer matrix $\mathbf{U}^{1} \mathbf{T}^{N} \mathbf{U}$ are defined as $\mathbf{S}^{N}=$ $\operatorname{diag}\left(\rho_{1}^{N}, \ldots, \rho_{h}^{N}\right), \mathbf{C}^{N}$ is the $2 n_{c}$ submatrix with diagonal $(2 \times 2)$ blocks, $\mathbf{C}_{j}^{N}, 1 \leqslant j \leqslant n_{c}$, given by

$$
\mathbf{C}_{j}^{N}=\rho_{j}^{N}\left[\begin{array}{cc}
\cos N \theta_{j} & \sin N \theta_{j} \\
\sin N \theta_{j} & \cos N \theta_{j}
\end{array}\right],
$$

and $\mathbf{P}^{N}$ is a matrix equal to $\mathbf{C}^{N}$ with $\rho=1$. In Eq. (11) symbols $\mathbf{S}, \mathbf{C}$ and $\mathbf{P}$ refer to submatrices listing eigenvalues belonging to stop, complex and pass bands respectively. The real wave vectors entering Eqs. (10) and (11), whose complex counterparts enter Eqs. (A.1) and (A.2) in Appendix A, represent the main computational difference between the proposed wave vector approach and the traditional one.

Eq. (11) can be rearranged to follow the real wave propagation direction,

$$
\left(\begin{array}{c}
\mathbf{r}_{B} \\
\mathbf{l}_{A}
\end{array}\right)=\left[\begin{array}{cc}
\boldsymbol{\Lambda}_{r}^{N} & \mathbf{0} \\
\mathbf{0} & \boldsymbol{\Lambda}_{l}^{N}
\end{array}\right]\left(\begin{array}{l}
\mathbf{r}_{A} \\
\mathbf{l}_{B}
\end{array}\right),
$$

where $\boldsymbol{\Lambda}_{r}^{N}=\operatorname{diag}\left(\mathbf{S}^{N}, \mathbf{C}^{N}, \mathbf{P}^{N}\right), \mathbf{\Lambda}_{l}^{N}=\operatorname{diag}\left(\mathbf{S}^{N}, \mathbf{C}^{N}\right), \mathbf{r}_{H}=\left(\mathbf{r}_{H}^{r}, \mathbf{r}_{H}^{c}, \mathbf{r}_{H}^{u}\right)$ and $\mathbf{l}_{H}=\left(\mathbf{l}_{H}^{r}, \mathbf{l}_{H}^{c}\right), H=$ $A, B$. It should be noticed that submatrices $\mathbf{P}^{N}$ are not rearranged, since they are not affected by ill-conditioning, thanks to the unit modulus of their eigenvalues. Therefore, rightward propagation of the associated $\mathbf{r}^{u}$ waves is considered, differently from the complex wave method.

The boundary conditions at the ends of the periodic structure expressed in terms of wave coordinates read as

$$
\mathbf{R}_{H} \mathbf{r}_{H}+\mathbf{L}_{H} \mathbf{l}_{H}=\mathbf{B}_{H} \mathbf{f}_{H}, \quad H=A, B,
$$

where $\mathbf{B}_{H}$ are Boolean matrices and $\mathbf{R}_{H}$ and $\mathbf{L}_{H}$ are $n \times\left(n_{r}+n_{c}+2 n_{u}\right)$ and $n \times x\left(n_{r}+n_{c}\right)$ rectangular submatrices of $\mathbf{U}$, respectively, varying according to the type of constraint and $\mathbf{f}_{H}$ are assigned ends' forces and/or displacements. Substituting eventually Eqs. (13) into (14) leads to the non-homogeneous problem

$$
\left[\begin{array}{cc}
\mathbf{R}_{A} & \mathbf{L}_{A} \boldsymbol{\Lambda}_{l}^{N} \\
\mathbf{R}_{B} \boldsymbol{\Lambda}_{r}^{N} & \mathbf{L}_{B}
\end{array}\right]\left(\begin{array}{c}
\mathbf{r}_{A} \\
\mathbf{l}_{B}
\end{array}\right)=\left(\begin{array}{c}
\mathbf{B}_{A} \mathbf{f}_{A} \\
\mathbf{B}_{B} \mathbf{f}_{B}
\end{array}\right) .
$$

The wave co-ordinates at the intermediate nodes can be derived and transformed back to obtain the response expressed by state variables; next, the response of the elements is readily evaluated as a function of the ends' displacements. However, while in the complex wave vectors approach the real state vectors are obtained by summing up products of complex conjugate eigenvectors and 
amplitudes, in the real wave vectors approach, the real state vectors are obtained by taking straight summation over products of real eigenvectors and amplitudes. Since the real eigenvectors represent the real and imaginary parts of the complex ones, the associated real amplitudes are half the real and imaginary parts of the complex amplitudes.

Similar to the complex wave formulation, the real one can be readily extended to piecewise periodic structures by introducing the scattering matrix arising at the interface of dissimilar cells as outlined in Ref. [9].

\section{A simple illustrative example}

In this section the proposed procedure is illustrated for a spring mass chain (mono-coupled system) already studied in Ref. [10] and shown in Fig. 2(a). It represents the discrete counterpart of the problem discussed in Section 2. By considering the spring mass chain composed by $N$ symmetric elements shown in Fig. 2(b), the transfer matrix reads as

$$
\mathbf{T}=\left[\begin{array}{cc}
\cos \vartheta & 1 \\
\sin ^{2} \vartheta & \cos \vartheta
\end{array}\right],
$$

where $\cos \vartheta=1 \quad \beta^{2}$ and $\sin \vartheta=\beta \sqrt{2} \quad \beta^{2}, \beta=(m / k)^{1 / 2} \omega$ being the frequency parameter. The transfer matrix eigenvalues are $\lambda_{1,2}=\mathrm{e}^{\mathrm{i} \mathrm{i} N \vartheta}$ and the pass band frequency range is $0 \leqslant \vartheta \leqslant \pi$. In the following subsections, the natural frequencies, modal shapes and forced response are determined by using several approaches, namely: transfer matrix, complex wave vectors and real wave vectors.

\subsection{Natural frequencies}

(a) Transfer matrix method. The transfer matrix of the whole chain is obtained by recursive product:

$$
\mathbf{T}^{N}=\left[\begin{array}{cc}
\cos N \vartheta & \frac{\sin N \vartheta}{\sin \vartheta} \\
\sin N \vartheta \sin \vartheta & \cos N \vartheta
\end{array}\right] .
$$

Then, by imposing the boundary conditions, the natural frequencies are obtained from the following equation:

$$
\sin N \vartheta \sin \vartheta=0, \quad \text { from which } \vartheta=\frac{j \pi}{N} \quad \text { with } j=0,1, \ldots, N \quad 1 .
$$

Eq. (18) is plotted in Fig. 3.

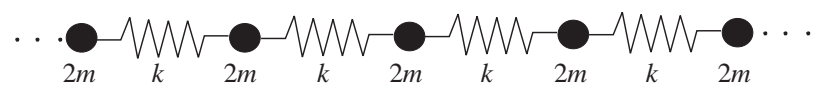

(a)

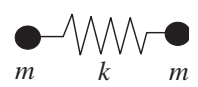

(b)

Fig. 2. Mono coupled system: (a) spring mass chain; (b) symmetric single unit. 


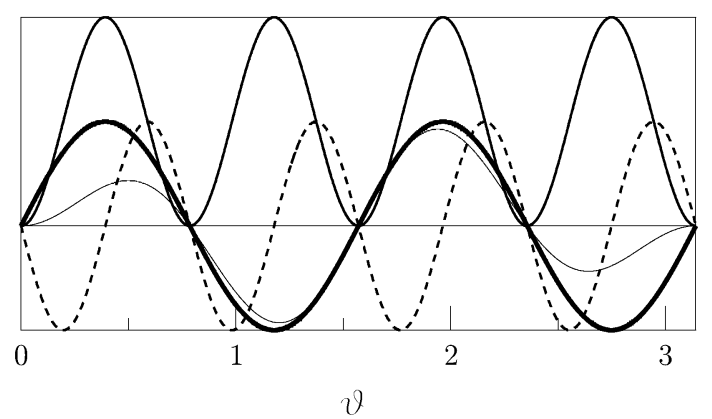

Fig. 3. Natural frequencies of the four bay spring mass system: (thin line) transfer matrix; (thick and dashed lines) real and imaginary parts of the complex wave vector method; (heavy line) real wave vector.

(b) Complex wave vector method. By applying Eq. (A.1), the complex wave vector method provides with the following state vector transformation:

$$
\left(\begin{array}{l}
d_{k} \\
f_{k}
\end{array}\right)=\left[\begin{array}{cc}
\frac{\mathrm{i}}{\sin \vartheta} & \frac{\mathrm{i}}{\sin \vartheta} \\
1 & 1
\end{array}\right]\left(\begin{array}{l}
r_{k} \\
l_{k}
\end{array}\right)
$$

Next, by imposing the boundary conditions (Eqs. (A.4) and (A.5)), a set of two equations is obtained:

$$
\left[\begin{array}{cc}
1 & \mathrm{e}^{\mathrm{i} N \vartheta} \\
\mathrm{e}^{\mathrm{i} N \vartheta} & 1
\end{array}\right]\left(\begin{array}{l}
r_{A} \\
l_{B}
\end{array}\right)=\left(\begin{array}{l}
0 \\
0
\end{array}\right) .
$$

The characteristic equation of the $2 \times 2$ boundary conditions' matrix is

$$
(\cos \vartheta+\mathrm{i} \sin \vartheta)^{2 N}=\cos 2 N \vartheta+\mathrm{i} \sin 2 N \vartheta=1 .
$$

The natural frequencies are given by the zeros of both its real and imaginary parts, represented in Fig. 3:

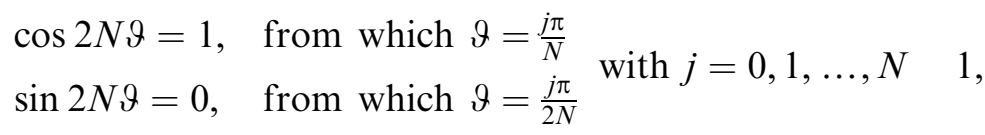

and therefore coincide with the roots of Eq. (18). It must be noted that real and imaginary parts of the characteristic equation cannot be individually zeroed, since while the former shows erroneous double roots, the latter has zeros that are not roots of the set. by

(c) Real wave vector method. According to Eq. (10), the state vector transformation is now given

$$
\left(\begin{array}{c}
d_{k} \\
f_{k}
\end{array}\right)=\left[\begin{array}{cc}
0 & \frac{1}{\sin \vartheta} \\
1 & 0
\end{array}\right]\left(\begin{array}{l}
r_{1, k} \\
r_{2, k}
\end{array}\right)
$$


By imposing the boundary conditions (Eqs. (14) and (15)), a set of two equations is obtained:

$$
\left[\begin{array}{cc}
1 & 0 \\
\cos N \vartheta & \sin N \vartheta
\end{array}\right]\left(\begin{array}{l}
r_{1, A} \\
r_{2, A}
\end{array}\right)=\left(\begin{array}{l}
0 \\
0
\end{array}\right) .
$$

The characteristic equation of the $(2 \times 2)$ boundary conditions' matrix, plotted in Fig. 3, and the natural frequencies are:

$$
\sin N \vartheta=0, \quad \text { from which } \vartheta=j \pi / N \text { with } j=0,1, \ldots, N \quad 1 .
$$

By summarizing, the example has shown the same issue discussed in Section 2. In particular, the complex wave vector method leads to a complex characteristic equation (of the type of Eq. (5)) in the real unknown $\omega$. When a numerical solution is required, computational problems arise; in contrast, the real wave vector method is not affected by these drawbacks.

\subsection{Modal shapes}

(a) Transfer matrix method. Modal shapes are derived by first setting $\mathbf{x}_{A}=(1,0)^{\mathrm{T}}$ and then obtaining $\mathbf{x}_{k}=\mathbf{T}^{k} \mathbf{x}_{A}$, from which the modal vector at the generic node $k$ reads

$$
\mathbf{x}_{k}=(\cos k \vartheta, \sin k \vartheta \sin \vartheta)^{\mathrm{T}} .
$$

(b) Complex wave vector method. From Eq. (20a), setting $r_{A}=1 / 2 \mathrm{i} \sin \vartheta, l_{B}=1 / 2 \mathrm{i} \sin \vartheta \mathrm{e}{ }^{\mathrm{i} N \vartheta}$ follows, and using rightward and leftward transmission, the complex wave co-ordinates at node $k$ are obtained

$$
r_{k}=r_{A} \mathrm{e}^{\mathrm{i} k \vartheta}, \quad l_{k}=l_{B} \mathrm{e}^{\mathrm{i}(N k) \vartheta} .
$$

Then, by substituting into Eq. (19), the state vector reads

$$
\left(\begin{array}{l}
d_{k} \\
f_{k}
\end{array}\right)=\left[\begin{array}{cc}
\frac{\mathrm{i}}{\sin \vartheta} & \frac{\mathrm{i}}{\sin \vartheta} \\
1 & 1
\end{array}\right]\left(\begin{array}{cc}
a_{k}+\mathrm{i} b_{k} \\
a_{k} & \mathrm{i} b_{k}
\end{array}\right),
$$

where

$$
a_{k}=\frac{1}{2} \sin \vartheta \sin k \vartheta, \quad b_{k}=\frac{1}{2} \sin \vartheta \cos k \vartheta,
$$

and result (26) is recovered. It must be noted that since the real state is expressed by a linear combination of two complex conjugate eigenvectors, the associated amplitudes must also be complex conjugate, i.e. $l_{k}=r_{k}$. Therefore the only independent quantities are the real $\left(a_{k}\right)$ and imaginary $\left(b_{k}\right)$ parts of $r_{k}$.

(c) Real wave vector method. From Eq. (24a), $r_{1, A}=0, r_{2, A}=\sin \vartheta$ and using rightward transmission, the real wave co-ordinates at node $k$ are obtained:

$$
\left(\begin{array}{l}
r_{1} \\
r_{2}
\end{array}\right)_{k}=\left[\begin{array}{cc}
\cos k \vartheta & \sin k \vartheta \\
\sin k \vartheta & \cos k \vartheta
\end{array}\right]\left(\begin{array}{l}
r_{1} \\
r_{2}
\end{array}\right)_{A}
$$


Then, by substituting into Eq. (23), the state vector expression reads as

$$
\left(\begin{array}{l}
d_{k} \\
f_{k}
\end{array}\right)=\left[\begin{array}{cc}
0 & \frac{1}{\sin \vartheta} \\
1 & 0
\end{array}\right]\left(\begin{array}{c}
2 a_{k} \\
2 b_{k}
\end{array}\right),
$$

where $a_{k}$ and $b_{k}$ are given by Eq. (29), and the resulting state vector $\mathbf{x}_{k}$ coincides with Eq. (26). In Eq. (31) the real state is expressed by a linear combination of two real eigenvectors, so that the associated amplitudes are also real and independent of each other. By comparing Eqs. (28) and (31) the relation between complex and real amplitudes, according to the two described approaches, can be readily deduced.

\subsection{Forced vibrations}

The forced response to a horizontal force applied at the left-hand boundary $A$ of the chain is determined.

(a) Transfer matrix method. From Eq. (6), with $\mathbf{T}^{N}$ given by Eq. (17), by imposing the boundary conditions $f_{A}=F$ and $f_{B}=0$, the state vector at the generic node $k$ reads as

$$
\mathbf{x}_{k}=\left(\frac{F \cos (N \quad k) \vartheta}{\sin \vartheta \sin N \vartheta}, \frac{F \sin (N \quad k) \vartheta}{\sin N \vartheta}\right)^{\mathrm{T}},
$$

(b) Complex wave vector method. Taking into account the force $F$ at the boundary $A$, Eqs. (20) become

$$
\left[\begin{array}{cc}
1 & \mathrm{e}^{\mathrm{i} N \vartheta} \\
\mathrm{e}^{\mathrm{i} N \vartheta} & 1
\end{array}\right]\left(\begin{array}{l}
r_{A} \\
l_{B}
\end{array}\right)=\left(\begin{array}{l}
F \\
0
\end{array}\right) .
$$

Then, by following the same steps described in the above section for deriving the modal shapes, an expression analogous to Eq. (28) is obtained, where

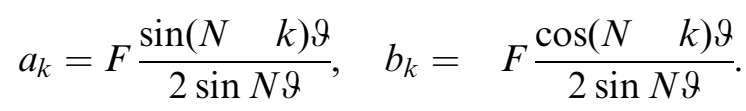

(c) Real wave vector method. Eqs. (24) modify:

$$
\left[\begin{array}{cc}
1 & 0 \\
\cos N \vartheta & \sin N \vartheta
\end{array}\right]\left(\begin{array}{l}
r_{1, A} \\
r_{2, A}
\end{array}\right)=\left(\begin{array}{c}
F \\
0
\end{array}\right) .
$$

By also following in this case the previously described steps, an expression analogous to (31) is obtained, in which $a_{k}$ and $b_{k}$ are given by Eqs. (34). Comments similar to the free response case hold.

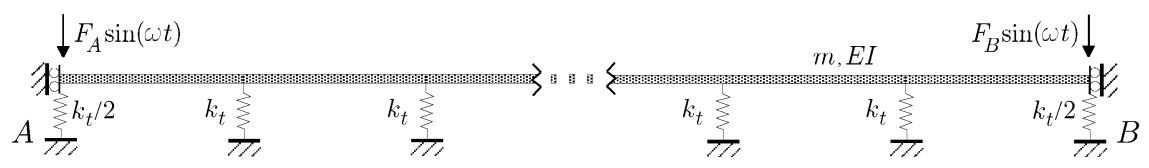

Fig. 4. Uniform Euler beam on evenly spaced springs. 


\section{Numerical results}

Bi-coupled periodic structures whose repetitive elements, as sketched in Fig. 4, are given by $N$ Euler beams resting on elastic supports with translational stiffness $k_{t} / 2$ and distributed mass $\mu$ are considered. The length and flexural stiffness of the beams are $l$ and $E I$ respectively. For such periodic elements, the state vector at the coupling point $k$ is given by $\mathbf{x}_{k}=\left(v_{k}, \varphi_{k}, V_{k}, M_{k}\right)^{\mathrm{T}}$, where $v, f$ and $V, M$ represent the generalized displacement and forces components respectively. The selected control parameters governing the propagation properties of a cell are the nondimensional frequency and spring stiffness defined as $\beta=\sqrt[4]{\mu \omega^{2}} l^{4} /(E I)$ and $\kappa=k_{t} l^{3} / E I$. Free wave propagation characteristics along the periodic structure are governed by the four eigenvalues of the transfer matrix $\mathbf{T}$. The regions where both the pairs of eigenvalues lie on the unit circle are referred to as pass pass (PP); the regions where only one pair of eigenvalues lies on the unit circle while the other pair is real are referred to as pass stop (PS); the regions where only real pairs of eigenvalues occur are the stop stop (SS) domains. Moreover, complex regions (C) exist where the eigenvalues are complex conjugate. In the pass regions waves propagate harmonically without attenuation, whereas in the stop regions waves decay; harmonic propagation with attenuation occurs in the complex regions. In Ref. [11], the boundaries of such propagation regions have been analytically derived on the plane of physical parameters $\kappa, \beta$. According to type of propagation regions, the real transfer matrix for wave vectors, defined by equation (11), takes the forms shown in Table 2.

Table 2

Global wave transfer matrix according to the propagation region

\begin{tabular}{lccc}
\hline Pass pass & Pass stop & Stop stop & Complex \\
\hline$\left[\begin{array}{cc}\mathbf{P}_{1}^{N} & \mathbf{0} \\
\mathbf{0} & \mathbf{P}_{2}^{N}\end{array}\right]$ & {$\left[\begin{array}{ccc}\lambda_{1}^{N} & \mathbf{0} & 0 \\
\mathbf{0} & \mathbf{P}_{1}^{N} & \mathbf{0} \\
0 & \mathbf{0} & \lambda_{1}^{-N}\end{array}\right]$} & {$\left[\begin{array}{cccc}\lambda_{1}^{N} & 0 & 0 & 0 \\
0 & \lambda_{2}^{N} & 0 & 0 \\
0 & 0 & \lambda_{2}^{-N} & 0 \\
0 & 0 & 0 & \lambda_{1}^{-N}\end{array}\right]$} & {$\left[\begin{array}{cc}\mathbf{C}_{1}^{N} & \mathbf{0} \\
\mathbf{0} & \mathbf{C}_{1}^{-N}\end{array}\right]$} \\
\end{tabular}
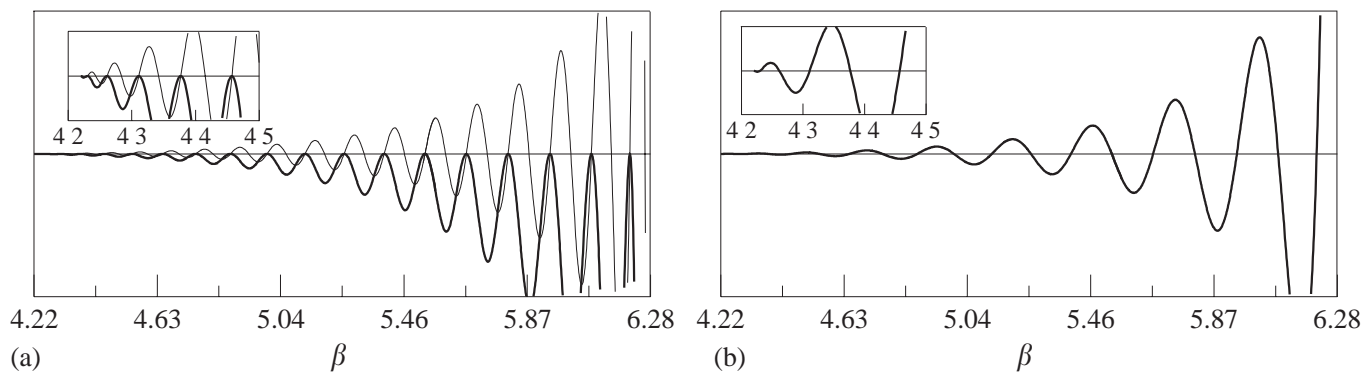

Fig. 5. Determinant of the boundary conditions' matrix for a 20 bay beam, $\kappa=113.75, \beta$ within the first PS: (a) complex wave vectors, (thick line) real part, (dashed line) imaginary part; (b) real wave vectors. 


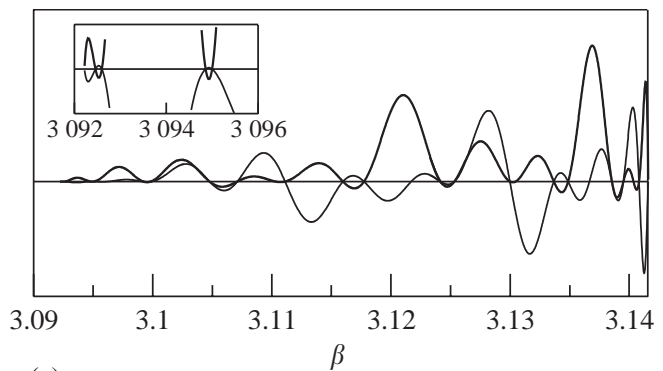

(a)

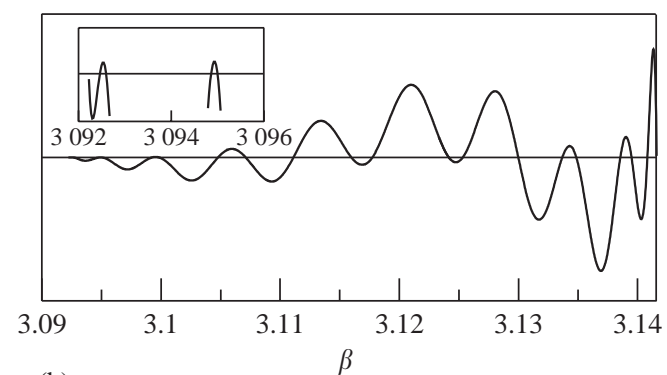

(b)

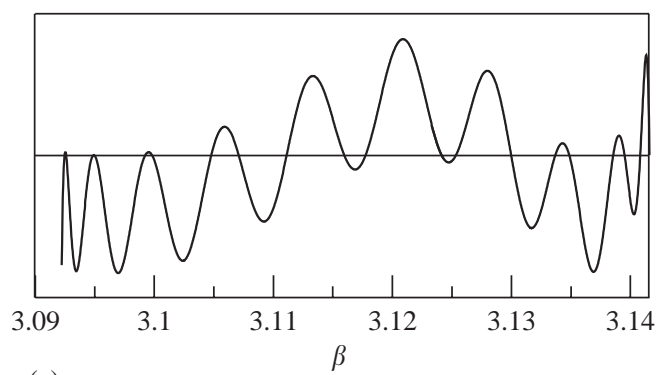

(c)

Fig. 6. Determinant of the boundary conditions' matrix for a 20 bay beam, $\kappa=113.75$, $\beta$ within the PP: (a) complex wave vectors, (thick line) real part, (dashed line) imaginary part; (b) real wave vectors; (c) transfer matrix.

\subsection{Free vibrations}

For symmetric bi-coupled elements it is well known that natural frequencies belong to PS and PP bands; moreover, within each band, they are as many as the number of elements composing the whole periodic structure. By zeroing the determinant of the system matrix in Eqs. (15), the natural frequencies of the bi-coupled periodic structure are obtained. In Figs. 5a and b the determinants of the system matrices for a 20-bay beam, with sliding boundary conditions at both ends, obtained through the complex and real wave vector approaches, respectively, are shown; the frequency range coincides with the first PS $(4.220 \leqslant \beta \leqslant 2 \pi)$ for $\kappa=113.75$. It must be noted that in the complex wave vector case (Fig. 5(a)), similar to the example of Section 4 (see Fig. 3), the natural frequencies occur whenever the real part of the determinant is tangent to the abscissa, thus implying a double root. This circumstance entails further computational difficulties. In contrast, the same roots are easily found in the real wave vector method (Fig. 5(b)). As expected, the same analysis carried out through the transfer matrix approach fails; indeed, numerical tests have shown that ill-conditioning occurs for beams with more than five elements. In Figs. 6(a) and (b) the determinants relevant to the unique PP band frequency range $(3.092 \leqslant \beta \leqslant \pi)$ are shown. Within this band the tangency to the $\beta$-axis of the real part observed in the PS band disappears and simultaneous crossings of both real and imaginary parts determine the natural frequencies (Fig. 6(a)). However, zeros of each function are found which are not root of the set (henceforth referred to as 'false' roots). Regardless of the number of elements, the transfer matrix illconditioning does not arise for frequencies belonging to PP bands, since its eigenvalues lie on the 

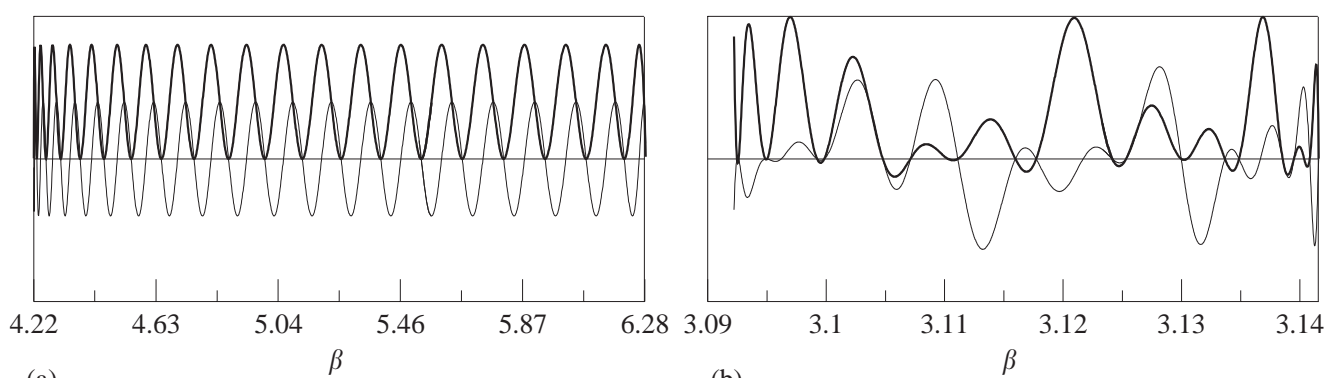

(a)

(b)

Fig. 7. Phase closure determinant for a 20 bay beam, $\kappa=113.75$ : (a) $\beta$ within the first PS, (thick line) real part, (dashed line) imaginary part; (b) $\beta$ within the PP.

unit circle; therefore the determinant of the 20-bay beam system matrix obtained through the transfer matrix approach also furnishes correct results (see Fig. 6(c)).

A further formulation, still involving complex wave propagation, has been proposed in literature to determine the natural frequencies of periodic structures [3]. It relies on the phase closure principle stating that natural frequencies occur when all wave modes complete a circumnavigation of the structure with a total phase change of $2 k \pi$. Accordingly, at an $N$-bay beam resonance, the following equation must be satisfied:

$$
\operatorname{det}\left[\boldsymbol{\Lambda}^{N} \mathbf{R}_{A}{ }^{1} \mathbf{L}_{A} \boldsymbol{\Lambda}^{N} \mathbf{L}_{B}{ }^{1} \mathbf{R}_{B} \quad \mathbf{I}\right]=\mathbf{0},
$$

where the matrices $\mathbf{R}_{H}, \mathbf{L}_{H}, H=A, B$, were introduced in the boundary conditions, Eq. (14), $\boldsymbol{\Lambda}$ is a $2 \times 2$ diagonal matrix collecting the eigenvalues with $|\lambda| \leqslant 1$ and $\mathbf{I}$ is the identity matrix. Fig. 7 is a plot of the determinant of Eq. (36) for the 20-bay beam so far considered. As expected, the results are analogous to those obtained with the complex wave vector method due to the common wave nature. Indeed, double roots of the real part occur in the first PS band (Fig. 7(a)) while false roots of both real and imaginary parts of the determinant are again found within the PP band (Fig. 7(b)). The errors reported in Ref. [3] for closely spaced natural frequencies did not take place in the present analysis in spite of frequency intervals twice as narrow $(\sim 0.0155 \mathrm{vs} . \sim 0.036 \mathrm{~Hz})$ within the PP band.

In Table 3 the first 40 natural frequencies of the 20-bay beam with sliding ends are reported; the bay beam was assumed to have a bending stiffness $E I=10 \mathrm{~N} \mathrm{~m}^{2}$, mass per unit length $\mu=$ $0.8 \mathrm{~N} / \mathrm{m}$, length $l=1 \mathrm{~m}$ and spring translational stiffness $k_{t}=1137.5 \mathrm{~N} / \mathrm{m}$. The natural frequencies obtained through both the wave vectors approaches are in perfect agreement and, as expected, the first 20 natural frequencies belong to the PP band $(9.56,9.87 \mathrm{~Hz})$ and the following 20 to the first PS $(17.81,39.48 \mathrm{~Hz})$. Moreover, due to the sliding ends' constraints, the last natural frequency in the PP band and the first natural frequency in the first PS band coincide with the PP right boundary and PS left boundary respectively. However, in the complex approach, both real and imaginary parts of the determinant of the system matrix must be considered due to false roots arising in both the PP and PS bands. In particular, within the PP band, false roots of the real and imaginary parts have been found at 9.67, 9.80, 9.86 Hz and 9.59, 9.74, $9.84 \mathrm{~Hz}$ respectively. As far as the first PS, false roots of the imaginary part occur every other true root, while all the natural frequencies determined by the real part correspond to double roots. Moreover, within the PP band, the natural frequencies obtained by the wave vector 
Table 3

Natural frequencies of the 20 bay beam with sliding sliding ends

\begin{tabular}{|c|c|c|c|c|}
\hline \multicolumn{2}{|c|}{ Wave vectors } & \multirow{2}{*}{$\frac{\text { Transfer matrix }}{9.56}$} & \multicolumn{2}{|c|}{ Finite element } \\
\hline 9.56 & 17.81 & & 9.59 & 12.74 \\
\hline 9.56 & 17.90 & 9.56 & 9.60 & 13.41 \\
\hline 9.58 & 18.16 & 9.58 & 9.62 & 15.53 \\
\hline 9.58 & 18.58 & 9.58 & 9.64 & 17.25 \\
\hline 9.60 & 19.16 & 9.60 & 9.66 & 18.42 \\
\hline 9.61 & 19.86 & 9.61 & 9.67 & 18.67 \\
\hline 9.64 & 20.69 & 9.64 & 9.71 & 19.72 \\
\hline 9.65 & 21.63 & 9.65 & 9.72 & 20.70 \\
\hline 9.68 & 22.66 & 9.68 & 9.74 & 21.98 \\
\hline 9.71 & 23.78 & 9.71 & 9.75 & 23.21 \\
\hline 9.72 & 24.99 & 9.72 & 9.76 & 24.44 \\
\hline 9.76 & 26.27 & 9.76 & 9.79 & 25.11 \\
\hline 9.77 & 27.63 & 9.77 & 9.80 & 28.08 \\
\hline 9.80 & 29.05 & 9.80 & 9.87 & 29.47 \\
\hline 9.82 & 30.54 & 9.82 & 9.87 & 31.06 \\
\hline 9.83 & 32.09 & 9.83 & 9.92 & 31.53 \\
\hline 9.85 & 33.69 & 9.85 & 10.00 & 33.45 \\
\hline 9.86 & 35.33 & 9.86 & 10.37 & 34.01 \\
\hline 9.86 & 36.99 & 9.86 & 11.53 & 36.88 \\
\hline 9.87 & 38.58 & 9.87 & 12.22 & 38.51 \\
\hline
\end{tabular}

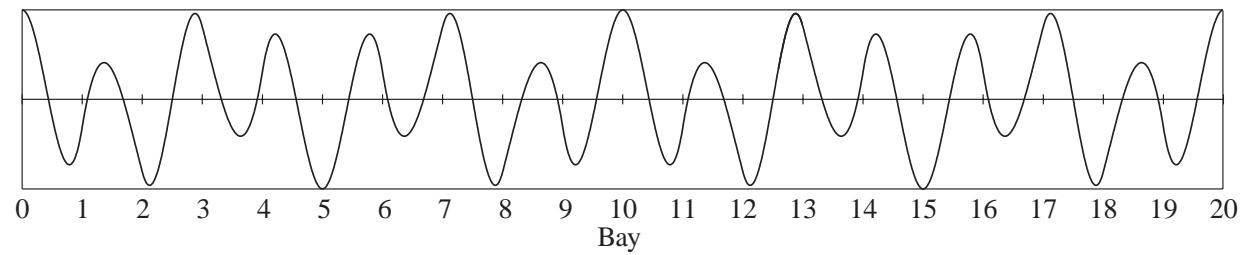

(a)

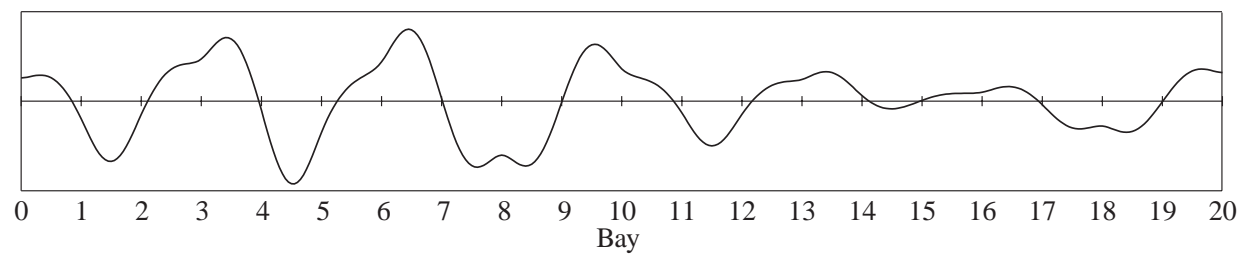

(b)

Fig. 8. First mode of the 20 bay beam: (a) wave vectors, $\omega=9.56 \mathrm{~Hz}$; (b) finite element, $\omega=9.59 \mathrm{~Hz}$.

methods coincide with those obtained through the transfer matrix. As expected, the latter approach fails inside the first PS band. The high modal density significantly affects the accuracy of the finite element results. Indeed, besides slight differences in most of the natural frequencies, few of them fall outside the PP and PS bands. The erroneous natural frequencies obtained through the finite element analysis are confirmed by the associated mode shapes violating the structural symmetry, as shown in Fig. 8 for the first mode. 


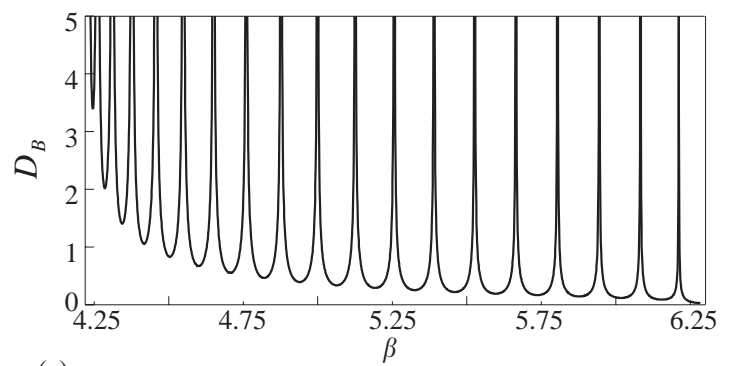

(a)

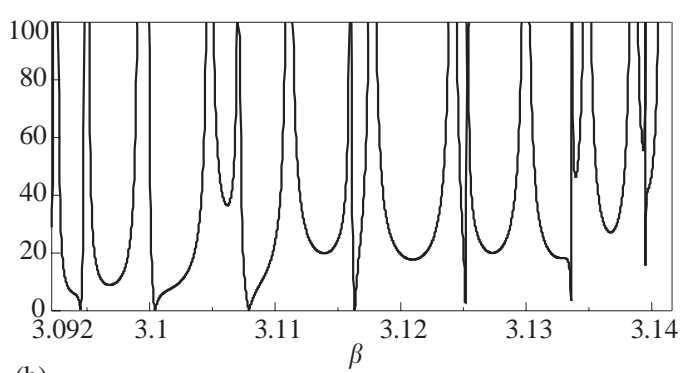

(b)

Fig. 9. Dynamic magnification factors at $B$ : (a) $\beta$ within the first PS; (b) $\beta$ within the PP.

\subsection{Forced vibrations}

The dynamics of the 20-bay beam has also been studied under a sweeping harmonic excitation applied at node $A$ (see Fig. 1). Fig. 9 shows the displacement dynamic magnification factors $D_{B}$ at node $B$ obtained through the real wave vectors approach. In particular, Fig. 9(a) refers to the response in the first PS band, while Fig. 9(b) refers to the PP band. For such an undamped structure the peaks of the resonance amplifications are ideally infinite. The different scales of both frequency and amplification ranges must be noticed.

\section{Conclusions}

A computational scheme, alternative to the well-known complex wave vector approach, has been proposed for dynamic analysis of long undamped periodic structures. The method has been suggested by an in-depth analysis of the source of some numerical difficulties encountered in literature in searching natural frequencies by the complex wave method. The cause has been ascribed here to the originally real problem being ill-posed, when it is recast in complex form. The new method has been conceived to take advantage of the main features of both transfer matrix and complex wave vectors approaches. Indeed, on one hand, the real transfer matrix for state vectors is transformed to a transfer matrix for wave vectors which is kept real, thus avoiding an ill-posed problem. On the other hand, transfer matrix ill-conditioning is avoided by using wave vectors arranged in order to let the computation proceed in the direction of wave motion. The method has been tested for a simple mono-coupled system and for a more complex structure. It has been found that the procedure is efficient and overcomes the methodological and numerical difficulties exhibited by other methods. However, the method entails an algorithm adapting to the type of propagation band, not required by the complex wave vector approach, which is independent of the propagation bands.

\section{Acknowledgements}

This work was partially supported by the MIUR-COFIN 2001-2003 grant. 


\section{Appendix A. Complex wave vector approach}

The main steps of the complex wave vector approach $[1,2]$ are reported. The $2 n$ state vector at the generic point $k$ can be transformed to complex wave vector through the matrix $\mathbf{U}$, whose columns are complex eigenvectors of $\mathbf{T}$, as follows:

$$
\left(\begin{array}{l}
\mathbf{d}_{k} \\
\mathbf{f}_{k}
\end{array}\right)=\mathbf{U}\left(\begin{array}{l}
\mathbf{r}_{k} \\
\mathbf{l}_{k}
\end{array}\right) \text {. }
$$

Substituting Eq. (A.1) into Eq. (6) gives

$$
\left(\begin{array}{l}
\mathbf{r}_{B} \\
\mathbf{l}_{B}
\end{array}\right)=\mathbf{U}{ }^{1} \mathbf{T}^{N} \mathbf{U}\left(\begin{array}{l}
\mathbf{r}_{A} \\
\mathbf{l}_{A}
\end{array}\right)=\left[\begin{array}{cc}
\boldsymbol{\Lambda}^{N} & \mathbf{0} \\
\mathbf{0} & \boldsymbol{\Lambda}
\end{array}\right]\left(\begin{array}{l}
\mathbf{r}_{A} \\
\mathbf{l}_{A}
\end{array}\right),
$$

where $\boldsymbol{\Lambda}$ is a diagonal matrix collecting the eigenvalues with $|\lambda| \leqslant 1$. Rearranging Eq. (A.2) to follow the waves propagation direction, it follows:

$$
\left(\begin{array}{l}
\mathbf{r}_{B} \\
\mathbf{l}_{A}
\end{array}\right)=\left[\begin{array}{cc}
\boldsymbol{\Lambda}^{N} & \mathbf{0} \\
\mathbf{0} & \boldsymbol{\Lambda}^{N}
\end{array}\right]\left(\begin{array}{l}
\mathbf{r}_{A} \\
\mathbf{l}_{B}
\end{array}\right),
$$

Eqs. (A.2) and (A.3) represent the transformation of the real transfer matrix for state vectors to the complex transfer matrix for wave vectors. The boundary conditions at the ends of the periodic chain in terms of wave co-ordinates read as

$$
\mathbf{R}_{H} \mathbf{r}_{H}+\mathbf{L}_{H} \mathbf{l}_{H}=\mathbf{B}_{H} \mathbf{f}_{H} \quad H=A, B,
$$

where $\mathbf{B}_{H}$ are Boolean matrices while $\mathbf{R}_{H}$ and $\mathbf{L}_{H}$ are square submatrices of $\mathbf{U}$ varying according to the type of constraint and $\mathbf{f}_{H}$ are assigned ends' forces and/or displacements. The core of the computational scheme consists of solving the sets of equations (A.3) and (A.4) by condensing the whole unknown wave vector in the sole components entering the domain. Using Eqs. (A.3) to eliminate the outgoing waves at the ends, the resolving equations (A.4) become

$$
\left[\begin{array}{cc}
\mathbf{R}_{A} & \mathbf{L}_{A} \boldsymbol{\Lambda}^{N} \\
\mathbf{R}_{B} \mathbf{\Lambda}^{N} & \mathbf{L}_{B}
\end{array}\right]\left(\begin{array}{c}
\mathbf{r}_{A} \\
\mathbf{l}_{B}
\end{array}\right)=\left(\begin{array}{c}
\mathbf{B}_{A} \mathbf{f}_{A} \\
\mathbf{B}_{B} \mathbf{f}_{B}
\end{array}\right) .
$$

From the knowledge of the wave co-ordinates at the boundaries $A$ and $B$, the response expressed by state variables is derived. The homogeneous form of problem (A.5) furnishes the natural frequencies and the 'mode' $\left(\mathbf{r}_{A}, \mathbf{l}_{B}\right)^{\mathrm{T}}$.

\section{References}

[1] A.H. von Flotow, Disturbance propagation in structural networks, Journal of Sound and Vibration 106 (1986) 433450.

[2] Y. Yong, Y.K. Lin, Propagation of decaying waves in periodic and piecewise periodic structures of finite length, Journal of Sound and Vibration 129 (1989) 99118.

[3] J. Signorelli, A.H. von Flotow, Wave propagation, power flow, and resonance in a truss beam, Journal of Sound and Vibration 126 (1988) 127144. 
[4] Y. Yong, Y.K. Lin, Dynamic response analysis of truss-type structural networks: a wave propagation approach, Journal of Sound and Vibration 156 (1992) 27-45.

[5] G.Q. Cai, Y.K. Lin, Wave propagation and scattering in structural networks, Journal of Engineering Mechanics 117 (1991) 1555-1574.

[6] D. Bouzit, C. Pierre, Wave localization and conversion phenomena in multi-coupled multi-span beams, Chaos, Solitons and Fractals 11 (2000) 1575-1596.

[7] F. Romeo, A. Luongo, Vibration reduction in piecewise bi-coupled periodic structures, Journal of Sound and Vibration 268 (2003) 601-615.

[8] W.J. Chen, C. Pierre, Exact linear dynamics of periodic and disordered truss beams: localization of normal modes and harmonic waves, Proceedings of 32nd AIAA/ASME/ASCE/AHS/ASC Structure, Structural Dynamics, and Material Conference, Baltimore, MD, April 1991.

[9] A. Luongo, F. Romeo, Real wave vectors for dynamic analysis of periodic structures, Eighth International Conference on Recent Advances in Structural Dynamics, ISVR University of Southampton, Southampton, 14-16 July 2003.

[10] M.G. Faulkner, D.P. Hong, Free vibrations of mono-coupled periodic system, Journal of Sound and Vibration 99 (1985) 29-42.

[11] F. Romeo, A. Luongo, Invariant representation of propagation properties for bi-coupled periodic structures, Journal of Sound and Vibration 257 (2002) 869-886. 